\title{
Exploiting the Plasticity of Primary and Secondary Response Mechanisms in Artificial Immune Systems
}

\author{
Julie Greensmith \\ School of Computer Science \\ University of Nottingham \\ Jubilee Campus \\ Nottingham, UK \\ jqg@cs.nott.ac.uk
}

\author{
Andrew M. Jackson \\ School of Medicine \\ University of Nottingham \\ City Hospital Campus \\ Nottingham, UK \\ andrew.jackson@nottingham.ac.uk
}

\author{
Ian Spendlove \\ School of Medicine \\ University of Nottingham \\ City Hospital Campus \\ Nottingham, UK \\ ian.spendlove@nottingham.ac.uk
}

\begin{abstract}
One of the key properties of the human immune system is to detect the presence of pathogens, and as such there are numberous immune algorithm which perform anomaly detection and pattern recognition. An additional facet of the human immune system is that an appropriate effector response is generated upon the detection of a pathogen - a process termed the primary response. Additionally the human immune system has the ability to remember the appropriate response to a particular pathogen - the secondary response. The complex orchestration of both the primary and secondary responses are highly dynamic - described in immunological terms as plastic. In this paper we present an overview of the the exact mechanisms of the generation of a T-helper cell primary response and the mechanisms by which it instructs secondary responses and discuss how this can be computationally useful in artificial immune system development.
\end{abstract}

\section{Keywords}

Artificial Immune Systems; Primary and Secondary Response

\section{INTRODUCTION}

Artificial immune systems are a collection of algorithms based on the function and behaviour of the human immune system. The majority of AIS focus on the detection of anomalies or optimisation of detection. AIS have directly employed primary and secondary response mechanisms. Timmis et al. [7] and Hunt and Cooke [3] incorporated it into a B-cell simulation model to construct one of the first immune network approaches with memory. Security based research by Sarafijanovic and Le Boudec [6] implemented a version of secondary response, producing a computationally faster response to previously encountered threats in data patterns in monitored mobile ad-hoc networks. Recently, interest in primary and secondary responses has diminished, potentially with the assumption that this component is uninteresting.

Permission to make digital or hard copies of part or all of this work for personal or classroom use is granted without fee provided that copies are not made or distributed for profit or commercial advantage and that copies bear this notice and the full citation on the first page. Copyrights for third-party components of this work must be honored. For all other uses, contact the owner/author(s).

GECCO'16 Companion July 20-24, 2016, Denver, CO, USA

(C) 2016 Copyright held by the owner/author(s).

ACM ISBN 978-1-4503-4323-7/16/07.

DOI: http://dx.doi.org/10.1145/2908961.2908962
The underlying immune mechanisms of primary and secondary response is coordinated by a class of white blood cell termed a T-helper cell. These cells have the ability to adapt to novel threats, termed pathogens, and to create an appropriate response. Depending on the type of response generated, the response is 'saved' in a memory system should the same pathogen be encountered in the future. The type of response memorised by the immune system is dependent on the nature of the primary T-helper cell response. The orchestration of a tightly regulated and refined primary response is based on the dynamic process of T-helper cell subtype switching. In this paper we present a summary of the T-helper cell mechanisms which create this dynamic primary response and how this generates and maintains a memory of the response for providing future protection. These mechanisms go beyond what has been used previously in AIS as it views the process of creating a secondary response from a primary response as a dynamic mechanism, containing numerous regulatory components which maintain the accuracy of the system.

\section{PLASTIC T-CELLS AND RESPONSES}

\subsection{Diversity in T-cells}

In terms of understanding T-helper cell dynamics, the term 'plasticity' refers to the ability of a T-cell to adopt different functional roles and behaviours under different environmental conditions. In immunological terms, the notion of T-cell plasticity is relatively new, and there is still debate and conjecture as to what defines the plastic T-cell subtypes. It may seem like trivial semantics, but the discovery that there is a difference between a cell lineage and a cell subtype is what has driven research in the understanding of the plasticity of T-cell responses[1]. The plasticity is thought to be able to create appropriate and tailored responses to both encountered and un-encountered pathogens.

Initially it was assumed that T-cell differentiation was restricted to a terminally differentiated lineage. In the $1980 \mathrm{~s}$ it was determined in mice models that T-helper cells differentiate into two classes of different functional subtypes namely T-helper type 1 (Th1) cells and T-helper type 2 (Th2) cells[4]. In strict laboratory conditions with mice with no prior pathogenic exposure, this mechanism is very clear. The Th1 and Th2 expressed different cytokine profiles, where a cytokine is defined as a molecular marker which is used in intracellular communication. In making this discovery, it was at the time, assumed that once a T-cell has 
committed to being a particular lineage, that this is fixed and there is no switching between different functional roles, as per the definition of a lineage. This model when tested in humans was not reproducible, it was not as clear cut as bacterial infections drive Th1 responses and allergenic conditions drive purely Th2 responses. The reality of the situation is far more sophisticated and complex. Evidence has now amassed to suggest that not only is T-cell subtype switching occurring is not an irregular event in exceptional circumstances, but is the normal behaviour in the majority of immune responses [1].

\subsection{Regulatory T-cells}

In AIS we have largely ignored is that T-cells also have a mechanism of suppression and tolerance in the form of regulatory T-cells. The interplay and the dynamics between pro-inflammatory Th1 or Th17 cells and that of regulatory T-cells (Treg) shows the production of a primary response to pathogens as more sophisticated than was first understood. As with the effector cells described above, different subtypes of Treg cell exist and perform different roles in the duration of the immune response. There are two main types of Treg cell, natural Tregs (nTregs) and inducible Tregs (iTregs).

The nTregs perform a crucial role in maintaining homeostasis and are responsible in part for the prevention of autoimmunity. In AIS we have viewed thymic selection from a purely negative selection perspective. However positive selection also occurs, and at the intersection of positive and negative selection, we find the formation of nTregs. Initially the iTreg is of another subtype, either a Th1 or Th17 cell. Changes in the context of the locale from a pro-inflammatory to an anti-inflammatory environment. The presence of IL10 is currently believed to be the key molecule involved in dynamically transforming T-cells to and from the iTreg subtype. When an infection occurs, the innate immune system translates both signals and antigen to the effector T-cell populations in lymph nodes. Given sufficient activation of the T-cells an adaptive response is mounted and several mechanisms initiate, including the production of antibodies and the recruitment and activation of innate cells [1].

\subsection{Dynamic Regulation of Memory}

The subtypes of T-cell created and maintained during a response also influence the creation and maintenance of immune memory. Memory is defined as the capacity to store and recall information from previously encountered events, and to respond more quickly and aggressively than on the first encounter. Classical models of T-cell memory relied on the clonal expansion of terminally differentiated Th1 or Th2 cells to prime the T-memory cells for rapid proliferation in the case of a future encounter. The memory T-cells escape the apototic process of the removal of T-cell clones following the resolution of infection[2]. The determination of what Tcells become memory cells is determined by the strength of the T-helper response, shaped by the plasticity of the effector T-cells. The discovery of the power of nTregs and iTregs however has augmented this viewpoint. It is thought that not only are memory cells generated to effector T-cells but also to T-cells with regulatory functionality. This drastically changes the dynamics of a memory based system from only remembering pathogenic associated antigen but to remembering antigen which bound to regulatory cells.
Memory regulatory T-cells are a challenge to define as a 'true' memory cell as self antigen is expressed constantly within the body, and therefore the notion that memory only exists in the absence of the antigen was violated in the definition of these memory cells. However, now laboratory techniques have advanced, this became possible leading to the characterisation of a cell which produces long lived responses against self antigen. The generation and persistence of an iTreg can add to this repertoire allowing memory to adapt and to tolerate 'changing self', shown in particular in the changes which take place during pregnancy. Evidence exists to show that in contrast to the nTregs the regulatory T-memory cells are equipped with extreme suppressive power[5]. While the exact mechanisms are an active area of research, what is interesting is that memory is not simply remembering the 'bad' but also being able to actively suppress autoimmune responses. In particular, regulatory T-memory cells are found in the periphery and not as restricted to the lymph node areas. The idea of duality in memory of cells, driven by the plasticity of the primary response, to actively maintain and promote tolerance and to provide an efficient basis for the maintenance of homeostasis.

\section{CONCLUSION}

Recent advances in epigenetic technqniques and phenotyping showed T-helper cell behaviour to be plastic. This will provide a fertile area of inspiration for creating novel 'plastic AIS' which are dually equipped with detection and response capabilitie and the ability to dynamically generate and maintain memory. Primary and secondary responses have been ignored in AIS for some time, but we hope that a fresh examination of these mechanisms will lead to improved novel AIS which can have a lasting impact in the development of novel AIS.

\section{REFERENCES}

[1] J. Bluestone, C. Mackay, J. O'Shea, and B. Stockinger. The functional plasticity of T cell subsets. Nature Reviews Immunology, 9(11):811-816, 2009.

[2] D. Farber. T cell memory: heterogeneity and mechanisms. Clinical Immunology, 95(3):173-181, 2000.

[3] J. Hunt and D. Cooke. An adaptive, distributed learning system based on the immune system. In Systems, Man and Cybernetics, volume 3, pages 2494-2499. IEEE, 1995.

[4] K. Murphy, P. Travers, and M. Walport. Janeway's Immunobiology. Garland Science, 7th edition, 2008.

[5] M. Rosenblum, S. Way, and A. Abbas. Regulatory t cell memory. Nature Reviews Immunology, 16:90-100, 2016.

[6] S. Sarafijanovic and J. Le Boudec. An artificial immune system for misbehavior detection in mobile ad-hoc networks with virtual thymus, clustering, danger signal and memory detectors. In Proc. of the 3rd International Conference on Artificial Immune Systems (ICARIS), LNCS 3239, pages 342-356, 2004.

[7] J. Timmis, M. Neal, and J. Hunt. An artificial immune system for data analysis. Biosystems, 55(1):143-150, 2000 . 